\title{
HYPERSPECTRAL IMAGE UNMIXING USING MANIFOLD LEARNING METHODS DERIVATIONS AND COMPARATIVE TESTS
}

\author{
Nguyen Hoang Nguyen ${ }^{(1)}$ Cédric Richard ${ }^{(1)}$ Paul Honeine ${ }^{(2)}$ Céline Theys $^{(1)}$ \\ (1) Laboratoire Lagrange, UMR 7293, Université de Nice Sophia-Antipolis, CNRS, OCA, France \\ (2) STMR, UMR 6279, Université de Technologie de Troyes, CNRS, France \\ nguyenh@oca.eu, cedric.richard@unice.fr, paul.honeine@utt.fr, celine.theys@unice.fr
}

\begin{abstract}
In hyperspectral image analysis, pixels are mixtures of spectral components associated to pure materials. Although the linear mixture model is the mostly studied case, nonlinear techniques have been proposed to overcome its limitations. In this paper, a manifold learning approach is used as a dimensionality-reduction step to deal with non-linearities beforehand, or is integrated directly in the endmember extraction and abundance estimation steps using geodesic distances. Simulation results show that these methods improve the precision of estimation in severely nonlinear cases.
\end{abstract}

\section{INTRODUCTION}

In hyperspectral imagery, a pixel is a mixture of spectral components associated with a number of pure materials present in the scene. In order to reveal underlying information, one needs to identify the endmembers present in the measured mixed pixels and derive the relative proportions of these materials. Under the assumption that the endmembers have been determined a priori using some off-the-shelf extraction techniques, see [2, 3, 4, 5], unmixing of hyperspectral images then consists of estimating their abundances [6]. In [7], these two problems are combined using a geometric formulation. The abundances are defined as barycentric coordinates in a reduced-dimensional space, and can be derived using either a ratio of simplex volumes, or a ratio of distances.

Most existing approaches are focused on linear models due to their inherent simplicity from conceptual and implementational points of view. In real images, the interaction between materials can cause nonlinear effects that may influence the precision in abundance calculation, which can violate the sum-to-one constraint and the non-negativity constraint. A possible strategy is, first, to use nonlinear methods for dimensionality reduction, such as ISOMAP [8] and LLE[9], and then to apply usual linear methods on reduced data. An interesting example is the combined use of geodesic distances and N-FINDR in [10].

In this paper, we generalize the approach described in [10] thanks to the use of geodesic distances with the geometric formulations for abundance estimation proposed in [7]. This paper is organized as follows. Section 2 is dedicated to endmember extraction and abundance estimation using a geometric approach. Section 3 introduces a new framework based on manifold learning to perform nonlinear unmixing, i.e., endmember extraction and abundance estimation. Section 4 provides some experimental results. Some concluding remarks are presented in Section 5.

\section{ENDMEMBER EXTRACTION AND ABUNDANCE ESTIMATION: A GEOMETRIC FRAMEWORK}

\subsection{Basic linear mixing and abundance estimation}

The basic linear mixing model can be expressed as

$$
r=M \alpha+n
$$

with $\boldsymbol{n}$ a noise vector, $\boldsymbol{r}=\left[r_{1}, \ldots, r_{L}\right]^{\top}$ an $L$-by- 1 column pixel supposed to be a mixture of $R$ endmember spectra. Each column $\boldsymbol{m}_{i}$ of the $L$-by- $R$ matrix $M$ is the spectrum of an endmember, and $\boldsymbol{\alpha}$ is the $R$-by-1 vector of abundances.

In practice, the spectral dimension is reduced to $(R-1)$ in order that the following mixing model be satisfied

$$
\boldsymbol{r}=\sum_{i=1}^{R} \alpha_{i} \boldsymbol{m}_{i}
$$

subject to the equality constraint $\sum_{i=1}^{R} \alpha_{i}=1$ and inequality constraints $\alpha_{i} \geq 0$. Suppose that the endmembers $\boldsymbol{m}_{i}$ have been extracted using any off-the-shelf technique. We shall now give a direct geometric framework to estimate the abundances in the same low-dimensional space. See [7] for extensive details. Estimating $\boldsymbol{\alpha}$ in (2) subject to the sum-to-one constraint can be performed by solving the following system

$$
\left(\begin{array}{cccc}
1 & 1 & \ldots & 1 \\
\boldsymbol{m}_{1} & \boldsymbol{m}_{2} & \ldots & \boldsymbol{m}_{R}
\end{array}\right) \boldsymbol{\alpha}=\left(\begin{array}{l}
1 \\
\boldsymbol{r}
\end{array}\right)
$$


Using Cramer's rule, the solution can be written as

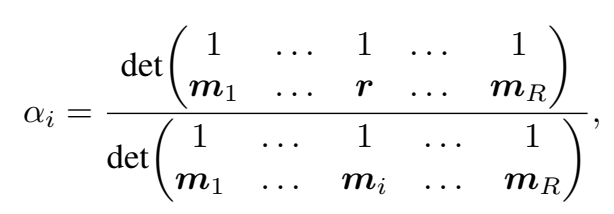

for all $i=1, \ldots, R$. This results can also be expressed by geometric formulation.

Consider the simplex $\mathcal{S}=\left\{\boldsymbol{m}_{1}, \boldsymbol{m}_{2}, \ldots, \boldsymbol{m}_{R}\right\}$. Its oriented volume can be calculated as follows

$$
\boldsymbol{V}_{\mathcal{S}}=\frac{1}{(R-1) !} \operatorname{det}\left(\begin{array}{cccc}
1 & 1 & \ldots & 1 \\
\boldsymbol{m}_{1} & \boldsymbol{m}_{2} & \ldots & \boldsymbol{m}_{R}
\end{array}\right)
$$

Let $\backslash$ denotes the set difference operator, $\mathcal{S} \backslash\left\{\boldsymbol{m}_{i}\right\} \cup\{\boldsymbol{r}\}$ is the set $\mathcal{S}$ with $\boldsymbol{m}_{i}$ replaced by $\boldsymbol{r}$. The calculation of each abundance coefficient $\alpha_{i}$ can thus be performed as

$$
\alpha_{i}=\frac{\boldsymbol{V}_{\mathcal{S} \backslash\left\{\boldsymbol{m}_{i}\right\} \cup\{\boldsymbol{r}\}}}{\boldsymbol{V}_{\mathcal{S}}}
$$

for all $i$. This can also be written in term of distances

$$
\boldsymbol{V}_{\mathcal{S}}=\frac{1}{(R-1) !} \delta\left(\boldsymbol{m}_{i}\right) \boldsymbol{V}_{\mathcal{S} \backslash\left\{\boldsymbol{m}_{i}\right\}}
$$

where $\delta\left(\boldsymbol{m}_{i}\right)$ is the signed distance between the vertex $\boldsymbol{m}_{i}$ and the subspace spanned by the other vertices of $\mathcal{S}$. This leads us to

$$
\alpha_{i}=\frac{\delta(\boldsymbol{r})}{\delta\left(\boldsymbol{m}_{i}\right)}
$$

for all $i$. As shown in [7], by using these equations, one can easily integrate abundance estimation into endmember extraction algorithms $[2,3,4,5]$.

\subsection{On non-negativity constraint violation}

The non-negativity constraint is violated if the pixel under consideration lies outside the simplex $\mathcal{S}$ formed by endmembers. In equations (6)-(8), volumes and distances are oriented. Their sign can be either positive or negative, depending on the order of the sequence defined by simplex vertices in the case of volume-based expressions, or on which side of the hyperplane the pixel lies in the case of distance-based expressions. Thus, equations (6)-(8) are appropriate for pixels that lie inside the simplex $\mathcal{S}$. A negative $\alpha_{i}$ means that the pixel is outside $\mathcal{S}$. An alternative approach, which lies beyond the scope of this paper, should be used to process these pixels.

\section{MANIFOLD LEARNING FOR ENDMEMBER EXTRACTION AND ABUNDANCE ESTIMATION}

The hypothesis model used by endmembers extraction methods and abundance estimation processes, such as those described above, are basically linear. Due to nonlinear physical effects such as multiple light scattering, this assumption may be uncorrect. Manifold learning can be used to overcome this drawback, by mapping the data into a lower-dimension manifold and returning the desired simplex. Manifold learning techniques considered hereafter are the basic ISOMAP [8] and LLE [9]. We shall now present some endmember extraction methods combined with abundance estimation that are based on manifold learning.

\subsection{N-FINDR}

The N-FINDR algorithm aims to find the endmembers by maximizing the volume of the simplex formed by pixels. The apices that define this simplex are the endmembers, provided that there exists a pure pixel for each endmember in the data set. A nonlinear dimensionality reduction is first performed with LLE or ISOMAP. The former maps data into a single global coordinate system of lower dimensionality, and can thus be used as a preprocessing step for endmember extraction and abundance estimation in this new coordinate system. As shown below, the latter can also be jointly used with equations (6) and (8) for endmember extraction and abundance estimation as it returns geodesic distances.

Volume calculation is usually accomplished by computing the determinant of a simplex matrix. It can also be written in terms of the Cayley-Menger determinant as shown independently in $[7,10]$. Let $d_{i j}$ be the Euclidean distance between endmember spectra $\boldsymbol{m}_{i}$ and $\boldsymbol{m}_{j}$. The square volume of the simplex defined by the $\boldsymbol{m}_{i}$ 's is given by

$$
\boldsymbol{V}_{\mathcal{S}}^{2}=\frac{(-1)^{R}}{2^{R-1}((R-1) !)^{2}} \operatorname{det}\left(\boldsymbol{C}_{1, \ldots, R}\right)
$$

with

$$
\boldsymbol{C}_{1, \ldots, R}=\left(\begin{array}{cc}
\boldsymbol{D}_{1, \ldots, R}^{2} & \mathbf{1} \\
\mathbf{1} & 0
\end{array}\right)
$$

where $\boldsymbol{D}_{1, \ldots, R}^{2}$ is the squared distance matrix whose $(i, j)$-th entry is $\boldsymbol{D}_{R}^{2}(i, j)=d_{i j}^{2}$ for all $i, j=1, \ldots, R$. Note that we can also rewrite (9) as

$$
\operatorname{det}\left(\boldsymbol{C}_{1, \ldots, R}\right)=-\left(\boldsymbol{d}_{1} \boldsymbol{C}_{2, \ldots, R}^{-1} \boldsymbol{d}_{1}^{\top}\right) \operatorname{det}\left(\boldsymbol{C}_{2, \ldots, R}\right)
$$

with $\boldsymbol{d}_{1}=\left(d_{12}^{2}, \ldots, d_{1 R}^{2}, 1\right)$. Finally, we can calculate the distance between the vertex $\boldsymbol{m}_{1}$ and the subspace spanned by the other vertices $\left(\boldsymbol{m}_{2}, \ldots, \boldsymbol{m}_{R}\right)$ as follows

$$
\delta\left(\boldsymbol{m}_{1}\right)=\left(\frac{\boldsymbol{d}_{1} \boldsymbol{C}_{2, \ldots, R}^{-1} \boldsymbol{d}_{1}^{\top}}{2}\right)^{\frac{1}{2}}
$$

Distances in equation (9) can be either Euclidean distances, or geodesic distances calculated with ISOMAP algorithm. This allows us to calculate $\boldsymbol{\alpha}$ via (6) or (8) based on manifold learning considerations. 


\subsection{SGA}

The SGA algorithm successively determines the endmembers as follows. First, the algorithm starts with a random point $\boldsymbol{t}$, and then searchs for another point that maximizes the volume of the simplex $\mathcal{S}_{1 i}=\left\{\boldsymbol{t}, \boldsymbol{r}_{i}\right\}$. This point is chosen as the first endmember and labeled $\boldsymbol{m}_{1}$. SGA continues with the simplexes $\mathcal{S}_{2 i}=\left(\boldsymbol{m}_{1}, \boldsymbol{r}_{i}\right)$. The point $\boldsymbol{r}_{i}$ that maximizes the volume of this simplex is selected as the second endmember and labeled $\boldsymbol{m}_{2}$. The searching process continues until the $R$ required endmembers are determined.

Using the Cayley-Menger determinant with geodesic distances calculated by ISOMAP leads to an improved SGA based on manifold learning. This algorithm jointly calculates volumes by using (9), and the abundances by using (6). LLE algorithm should also be used, but as a preprocessing stage for dimensionality reduction since it does not return geodesic distances. In this case, equations (6) and (8) can used for abundance estimation in this new coordinate system.

\subsection{VCA}

The VCA algorithm is based on the fact that the affine transformation of a simplex is also a simplex. It then projects the data onto a direction that is orthogonal to the subspace spanned by the endmembers that were found previously. The farthest point gives the new endmember. The algorithm iterates until it reaches the desired number of endmembers. Basically, VCA processes the distance between a point and the subspace spanned by the endmembers. Again, we can use geodesic distances returned by ISOMAP with (8) and (11) to get a manifold-learning based algorithm that jointly determines the endmembers and estimates the abundances.

\section{SIMULATION RESULTS}

We shall now compare all these algorithms using an artificial data set that consists of random samples in a two-dimensional simplex, transformed into a three-dimensional nonlinear manifold by projection on a swissroll structure. See [10] for details on the experimental setup. The non-linearity of the swissroll data is parameterized by a variable $\sigma$. The endmembers are also in the dataset. The coordinate of a data point $\boldsymbol{r}_{i}$ as a function of the abundance local $\boldsymbol{\alpha}_{i}$ can thus be expressed by

$$
\begin{aligned}
& r_{i 1}=\alpha_{i 1} \sin \left(\sigma \alpha_{i 1}\right)+1 \\
& r_{i 2}=\alpha_{i 1} \cos \left(\sigma \alpha_{i 1}\right)+1 \\
& r_{i 3}=\alpha_{i 2}+1
\end{aligned}
$$

Following the sum-to-one constraint, the abundance of the third endmembers can be generated by $\alpha_{i 3}=1-\left(\alpha_{i 1}+\alpha_{i 2}\right)$. By setting a single abundance to one, the others to zero, one can find the endmembers

$$
\begin{aligned}
& \boldsymbol{m}_{1}=(\sin (\sigma)+1, \cos (\sigma)+1,1) \\
& \boldsymbol{m}_{2}=(1,1,2) \\
& \boldsymbol{m}_{3}=(1,1,1)
\end{aligned}
$$

Swissroll data unmixing was performed over a 1000-sample set using the conventional and the manifold-learning based algorithms. The number of endmembers was set to $R=3$. Endmember extraction was performed with N-FINDR, SGA and VCA, in a linear and a nonlinear way. Linear processing consisted of performing first a PCA to map the data into a two-dimensional space, and then applying one of the above three algorithms. LLE and ISOMAP were considered for nonlinear processing. The former was used as a preprocessing step, prior to N-FINDR, SGA and VCA, which were jointly applied with (6) and (8) to extract endmembers and evaluate their fractions of abundance in mixed pixels. The latter was applied to compute the geodesic distances in (9)-(11), which were used by N-FINDR, SGA and VCA to extract the endmember, and to evaluate the abundances via (6) and (8).

These algorithms were compared for $\sigma$ values in $[0,5]$. The upper graphic in Figures 1-3 shows the mean of the minimum spectral angle between estimated endmembers $\widehat{\boldsymbol{m}}_{i}$ and the ground truth $\boldsymbol{m}_{i}$.

$$
\theta=\frac{1}{R} \sum_{i=1}^{R} \min _{j} \arccos \left(\frac{\widehat{\boldsymbol{m}}_{i} \cdot \boldsymbol{m}_{j}}{\left\|\widehat{\boldsymbol{m}}_{i}\right\|\left\|\boldsymbol{m}_{j}\right\|}\right)
$$

It can be observed that the nonlinear algorithms yield acceptable error values over the entire range of $\sigma$, and perform better than the linear algorithms. As the nonlinearity parameterized by $\sigma$ increases, they however fail to retrieve all the endmembers. The lower graphic in Figures 1-3 shows the error between the original and the estimated abundances defined by

$$
E=\frac{1}{R} \sum_{k=1}^{R} \min _{k^{\prime}}\left(\frac{1}{N} \sum_{i=1}^{N}\left|\widehat{\alpha}_{i k}-\alpha_{i k^{\prime}}\right|\right)
$$

where $N=1000$ is the size of the data set. Again, nonlinear algorithms have better performance than their linear counterparts. Figures 4-5 present the same results in a different way, which allows to compare the performance of N-FINDR, SGA and VCA algorithms when associated with ISOMAP and LLE, respectively. It appears that ISOMAP performs better than LLE, but needs more time-consuming calculations.

\section{CONCLUDING REMARKS}

In this paper, we studied several manifold-learning based approaches for joint endmember extraction and abundance estimation in hyperspectral images. Simulation showed that they perform better than usual linear approaches in nonlinear unmixing problems. These results were confirmed by experiments on well-known hyperspectral scenes, which will be presented in the camera-ready paper. 

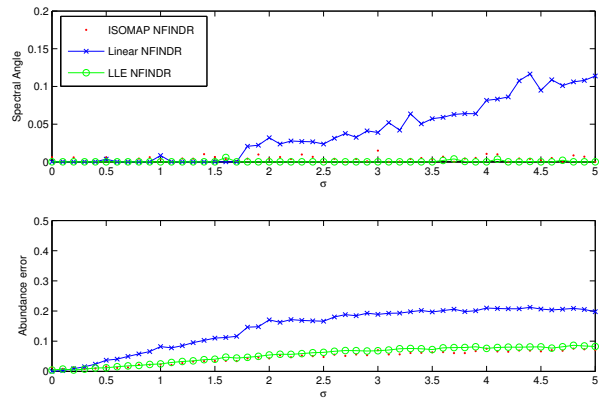

Fig. 1: N-FINR algorithm with PCA (linear), ISOMAP and LLE
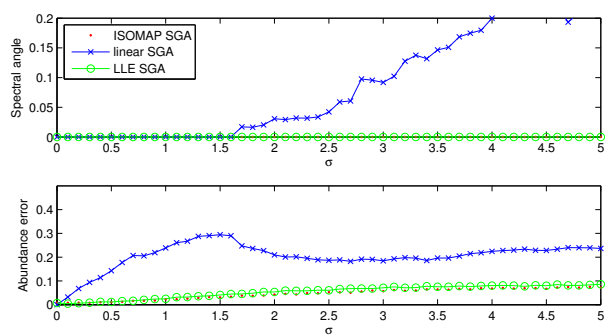

Fig. 2: SGA algorithm with PCA (linear), ISOMAP and LLE
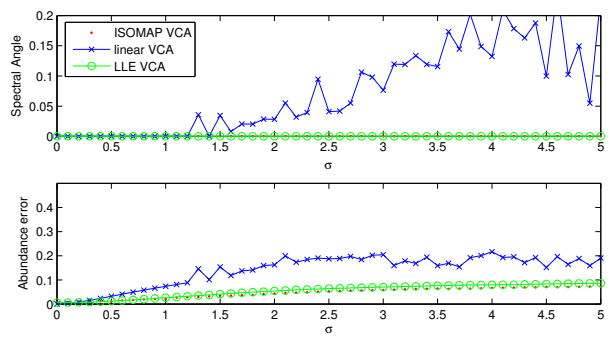

Fig. 3: VCA algorithm with PCA (linear), ISOMAP and LLE

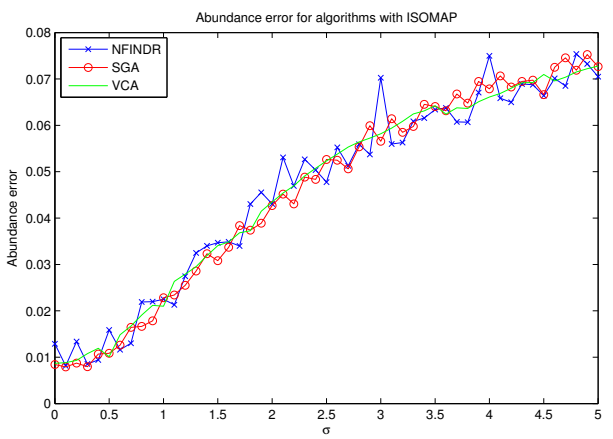

Fig. 4: Abundance error for N-FINDR, SGA and VCA when associated with ISOMAP

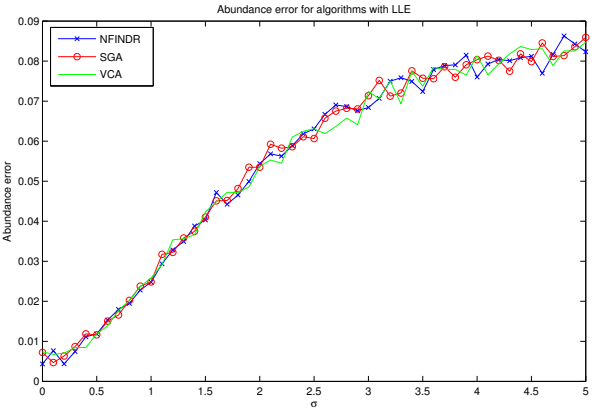

Fig. 5: Abundance error for N-FINDR, SGA and VCA when associated with LLE

\section{REFERENCES}

[1] N. Keshava and J. F. Mustard, "Spectral unmixing," IEEE Signal Processing Magazine, vol. 19, no. 1, pp. 44-57, 2002.

[2] M. E. Winter, "N-FINDR: an algorithm for fast autonomous spectral end-member determination in hyperspectral data," in Proc. SPIE, 1999, vol. 3753, pp. 266-277.

[3] C. I. Chang, C. C. Wu, W. Liu, and Y. C. Ouyang, "A new growing method for simplex-based endmember extraction algorithm," IEEE Transactions on Geoscience and Remote Sensing, vol. 44, no. 10, pp. 2804-2819, 2006.

[4] José M. P. Nascimento and José M. Bioucas-Dias, "Vertex component analysis: A fast algorithm to unmix hyperspectral data," IEEE Transactions on Geoscience and Remote Sensing, vol. 43, no. 4, pp. 898-910, April 2005.

[5] J. W. Boardman, "Geometric mixture analysis of imaging spectrometry data," in Proc. IEEE IGARSS, 1994, vol. 4, pp. 23692371.

[6] D. C. Heinz and C.-I Chang, "Fully constrained least squares linear mixture analysis for material quantification in hyperspectral imagery," IEEE Transactions on Geoscience and Remote Sensing, vol. 39, no. 3, pp. 529-545, 2001.

[7] P. Honeine and C. Richard, "Geometric unmixing of large hyperspectral images: A barycentric coordinate approach," IEEE Transactions Geoscience and Remote Sensing, pp. 1-11, November 2011.

[8] J. B. Tenenbaum, V. de Silva, and J. C. Langford, "A global geometric framework for nonlinear dimensionality reduction," Science, vol. 290, no. 5500, pp. 2319-2323, 2000.

[9] S. Roweis and L. Saul, "Nonlinear dimensionality reduction by locally linear embedding," Science, pp. 2323-2326, 2000.

[10] R. Heylen, D. Burazenoviv, and P. Scheunders, "Non-linear spectral unmixing by geodesic simplex volume maximization," IEEE Journal of Selected Topics in Signal Processing, vol. 5, no. 3, pp. 534-542, June 2011. 